\title{
Utilidad de la Biopsia de Ovario por Laparoscopia
}

\author{
Dres.: :Villiam Onatra H.*, Manuel Lagos G.** , Héctor Enrique Bernal*** \\ Ricardo Alvarado P.****
}

\section{INTRODUCCION}

En el estudio de las pacientes con problemas ginecológicos de orden endocrino $y$ de infertilidad se ha utilizado la técnica de laparoscopia para visualizar los órganos pélvicos y además para la toma de biopsia ovárica para complementar y confirmar el diagnóstico clínico (1), (2), (3). Al mejorar los procedimientos no sólo se pudieron visualizar estas estructuras sino que se idearon instrumentos técnicos para practicar pequerías cirugías como resección de quistes de ovario, miomas, embarazos ectópicos incipientes.

* Instructor Asociado del Dpto. de Obstetricia y Ginecología U.N.

** Instructor Asociado del Dpto. de Obstetricia y Ginecología. U.N.

*** Profesor Asociado del Dpto. de Obstetricia y Ginecología. U.N.

**** Profesor Asociado del Dpto. de Patología U.N.
Hasta hace unos años la función gonadal sólo se podía determinar por temperatura basal, biopsia endometrial, citología vaginal funcional y medición de esteroides en orina. Hoy gracias a la ecografía, al radioinmunoanálisis y a la manipulación técnica de los órganos pélvicos se pueden precisar mejor el diagnóstico tanto de la patología endocrina como de la infertilidad (4). La utilidad de la biopsia de ovario por laparoscopia ha sido cuestionada (5). Con base a que la muestra puede ser muy poco representativa a la hemorragia en el sitio de biopsia y a adherencias posteriores que complicarían el cuadro en las pacientes infértiles. A pesar de ésto en casos de disfunción ovárica como amenorrea primaria y secundaria ha prestado su apoyo no sólo diagnóstico sino que el clínico puede inferir sobre su pronóstico. Fue interés de los autores revisar y confirmar en nuestro medio la utilidad que puede prestar la biopsia ovárica por laparoscopia como parte de estudio de pacientes que consultaron en las clínicas de endocrinología e infertilidad, Departamento de Obstetricia y Ginecología de la Universidad Nacional en el Hospital Materno Infantil de Bogotá. 


\section{MATERIAL Y METODOS}

Se analizarón 87 procedimientos de laparoscopia diagnóstica llevados a cabo en el Hospital Materno Infantil de Bogotá de 1 de julio de 1979 al 31 de diciembre de 1980 . Se tomó biopsia ovárica en 40 casos $(45.9 \%$ ), de las cuales se practicaron en forma bilateral a 6 (15\%) y 34 (75\%) en unilateral teniendo en cuenta si existía una lesión patológica o no. La laparoscopia se llevo a cabo por incisión infraumbilical y un laparoscopio convencional. La biopsia se tomó con pinza de Palmer sin doble punción. En los casos de infertilidad se realizó el día $24 \mathrm{del}$ cíclo.

Para el diagnóstico laparoscópico se tuvo en cuenta, el tamaño, forma y características del útero; forma, color y permeabilidad de las trompas y en ovario su forma, diámetros aproximados, signos o no de ovulación; adhèrencias o no al piso pélvico, signos de endometriosis, descripción y características en los casos de hidrosalpin $x$ o quistes de ovario. La biopsia se tomó bajo visión directa teniendo en cuenta el borde antimesenterico y el ovario elegido en el que se sospechaba alguna patología. En los casos que se presentó hemorragía se utilizó el electrocauterio y cuidadosa revisión de la hemostasia. Una vez obtenido el especimen éste se llevó inmediatamente al laboratorio de patología para su fijación. Se procesó en cortes de parafina y la coloración utilizada fue hematoxilina eosina. En el estudio histológico se tuvo en cuenta el espesor de la corteza, presencia o no de fol ículos, actividad de ellos, presencia o no de cuerpo amarillo o blanco.

Con el fin de analizar la utilidad que pudiera prestar ésta biopsia de acuerdo a las diferentes entidades, se dividieron los casos en dos grandes grupos: Grupo l, Endocrinología subclasificado en amenorrea primaria, amenorrea secundaria y trastor- nos del ciclo menstrual. Grupo II, infertilidad subclasificándola en infertilidad primaria y secundaria. En los parámetros clínicos se tuvo en cuenta la edad, motivo de consulta y evolución de la enfermedad. Paraclínicamente se tuvo en cuenta la citología vaginal funcional, FSH, LH, Estradiol, PBI, Ginecografía en los casos de amenorrea y cariotipo en amenorrea primaria. En los estudios de infertilidad la histerosalpingografía, biopsia de endometrio fueron complementarios. Para la determinación de hormonas FSH, LH, Estradiol, se procesaron en el laboratorio de endocrinología del Hospital Militar Central. Los antisueros para hormonas gonadotropas fueron adquiridos a través de la agencia de la pituitaria del Instituto Nacional de Salud de los Estados Unidos. Lós trazadores se marcaron en el laboratorio con lodo 125 por el método de Cloryamina T. Para el radioinmunoensayo se utilizó el método del segundo anticuerpo y la lectura se realizó en un contador Gama marca Siemens. El Estradiol fue determinado de acuerdo a los patrones de Laboratorios Merck y el antisuero fue obtenido en el propio laboratorio. La extracción se hizo por el método de éter etílico y la separación de la fracción libre de la ligada por el método de Carbón dextrano. El trazador fue obtenido en la "New England Nuclear". La lectura se hizo en un contador Beta Beckman automático.

Los estudios de cariotipo se llevaron a cabo en la Sección de Genética de la UN. PBI en la Sección de Endocrinología Dpto. de Medicina de la UN. La histerosalpingografía $y$ ginecografía en la Sección de Radiología UN. Biopsia de endometrio en el Dpto. de Patología sección Materno Infantil.

Una vez completados los estudios y una vez propuesto un diagnóstico definitivo se instauró un tratamiento y los resultados fueron satisfactorios o no de acuerdo a la patología encontrada. 


\section{RESULTADOS}

De las 40 biopsias, 18 (45\%) correspondieron a consultas de endocrinología, 7 (17.5\%) a amenorrea primaria, 6 (15\%) amenorrea secundaria y $5(12.5 \%)$ a trastornos del ciclo, incluyendo aquí un caso especial de biopsia ovárica en una mujer menopáusica. El grupo de infertilidad aportó 22 casos, 15 (37.5\%) de causa primaria y $7(17.5 \%)$ de secundaria. Tabla No. 1.

Amenorrea Primaria: Tablas 2.a. - 2.b.

En los casos de amenorrea primaria encontramos que el promedio de edad fue de 18 años y a pesar de que en este grupo aparecen la hipoplasia útero ovárica como diagnóstico dominante (casos
Tabla No. 1

BIOPSIA DE OVARIO POR LAPAROSCOPIA

\begin{tabular}{|c|c|c|c|c|c|}
\hline Entiden & No. Casess & : & SUBCLASIFICACION & No. Conos & $z$ \\
\hline \multirow{3}{*}{ Endocrinologia } & \multirow{3}{*}{18} & \multirow{3}{*}{45.0} & Nemportes i? & , & 17.5. \\
\hline & & & Mmonotreas $2^{2}$ & 6 & 15.0 \\
\hline & & & ciecto istegutar & s & 12.5 \\
\hline \multirow{2}{*}{ Inferciliddd } & \multirow{2}{*}{22} & \multirow{2}{*}{35.6} & Primaria & is & 37.5 \\
\hline & & & Securdaria & 7 & 17.5 \\
\hline Tota: & 40 & $100:$ & & $\triangle 0$ & 100 \\
\hline
\end{tabular}

$4,5,6$ y 7) agenesia (casos 1 y 3) no se aprecia alteración del cariotipo. La determinación hormonal de $\mathrm{FSH}$ y $\mathrm{LH}$, Estradiol complementa el diagnóstico de una pobre función ovárica en los ca$\operatorname{sos} 4,5,6,7$, hipogonadismo hipogonadotropo $(1,3,6)$, hipogonadismo hiper-

Tabla No. 2.a.

AMENORREA 1 a.

\begin{tabular}{|c|c|c|c|c|c|c|c|c|}
\hline CASO & EDAD & $\begin{array}{l}\text { DIAGNOSTICO } \\
\text { INICIAL }\end{array}$ & $\begin{array}{l}\text { FSH } \\
\mathrm{mUI}\end{array}$ & $\begin{array}{c}\mathrm{LH} \\
\mathrm{mUI}\end{array}$ & $\begin{array}{c}\mathrm{E}_{2} \\
\mathrm{Pg} / \mathrm{m} /\end{array}$ & $\begin{array}{l}\mathrm{PBI} \\
\mathrm{mcg} \%\end{array}$ & C.V.F. & CARIOTIPO \\
\hline 1 & 18 & $\begin{array}{l}\text { Agenesia } \\
\text { utero-ovárica } \\
\text { Hipotiroidismo }\end{array}$ & 2.0 & 1.9 & 44.0 & 3.05 & $\begin{array}{c}80-20-10 \\
\left(c_{1}\right)\end{array}$ & $46 \mathrm{xx}$ \\
\hline 2 & 18 & $\begin{array}{l}\text { Poliquistosis } \\
\text { ovárica }\end{array}$ & 10.0 & 46.0 & 63.0 & 4.0 & $\begin{array}{c}10-90-0 \\
\left(\mathrm{C}_{2}\right)\end{array}$ & $46 \mathrm{xx}$ \\
\hline 3 & 19 & $\begin{array}{l}\text { Agenesia } \\
\text { utero-ovárica }\end{array}$ & 3.0 & 2.1 & 30.0 & 3.9 & $\begin{array}{c}90-10-0 \\
\left(C_{1}\right)\end{array}$ & $46 \mathrm{xx}$ \\
\hline 4 & 18 & $\begin{array}{l}\text { Agenesia } \\
\text { utero ovárica }\end{array}$ & 33.0 & 12.5 & $<12.5$ & 5.2 & $\begin{array}{c}10-90-0 \\
\left(c_{2}\right)\end{array}$ & $46 x x$ \\
\hline 5 & 20 & $\begin{array}{l}\text { Hipoplasia } \\
\text { utero ovárica } \\
\text { Hipotiroidismo }\end{array}$ & 18.0 & 42.4 & $<12.5$ & 3.5 & $\begin{array}{c}40-60-0 \\
\left(c_{2}\right)\end{array}$ & $46 \mathrm{XX}$ \\
\hline 6 & 18 & $\begin{array}{l}\text { Hipoplasia } \\
\text { utero-ovárica }\end{array}$ & 0.85 & 11.5 & $<12.5$ & 5.6 & $\begin{array}{c}20-80-0 \\
\left(C_{2}\right)\end{array}$ & $46 \mathrm{xX}$ \\
\hline 7 & 22 & $\begin{array}{l}\text { Agenesia } \\
\text { utero-cvárica }\end{array}$ & 76.5 & 35.0 & $<12.5$ & 7.2 & $\begin{array}{c}0-30-10 \\
\left(c_{2}\right)\end{array}$ & $46 \mathrm{xx}$ \\
\hline
\end{tabular}


Tabla No. 2.b.

AMENORREA 1a.

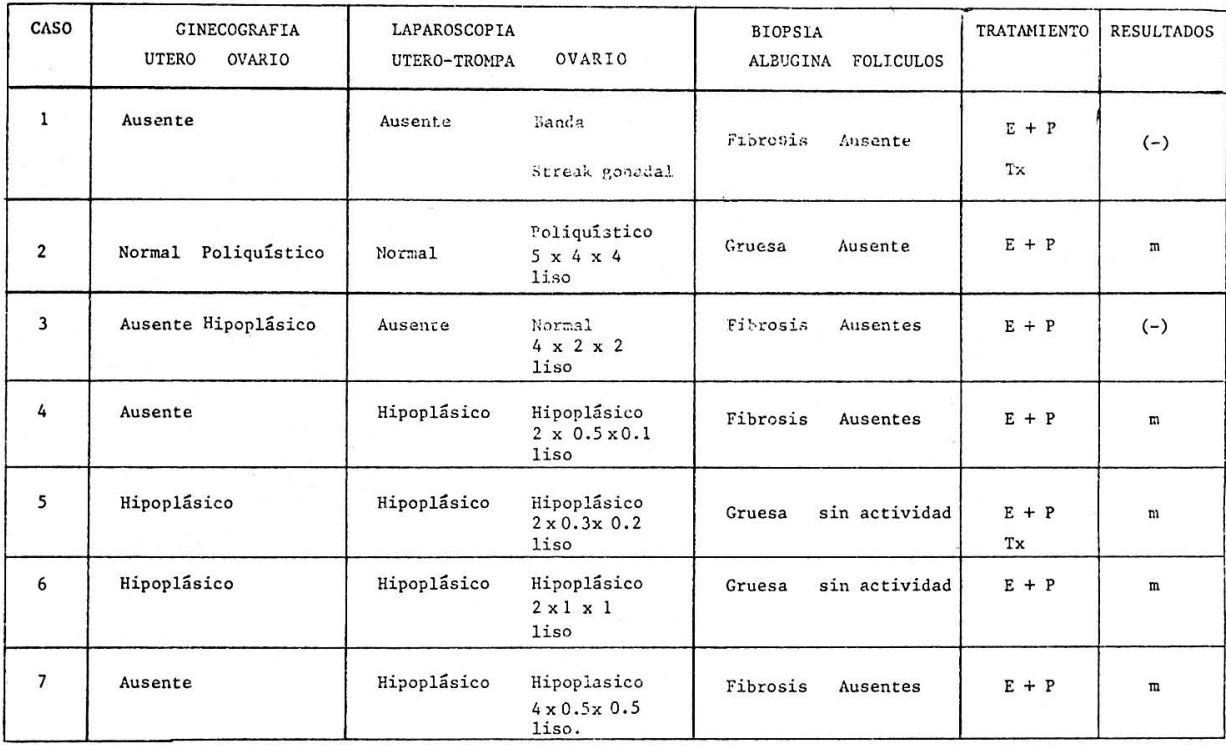

$\mathrm{E}$ = Estrógeno $\mathrm{P}=$ Progestágeno $\quad \mathrm{Tx}=$ Tiroides $\mathrm{m}$ a menstruaciốn.

gonadotropo (caso 7), hipogonadismo normogonadotropo $(4,5)$ y la confirmación de ovario poliquístico con elevación de la LH en el caso No. 2. La demostración de la asociación con hipotiroidismo en dos casos ( 1 y 5). En este grupo la citología vaginal confirma la escasa acción de los estrógenos.

En el pasado la ginecografía era un estudio casi obligado en el estudio pélvico de las pacientes amenorréicas. Si miramos la correlación directa frente a la laparoscopia encontramos que fue directa en 4 casos $(1,2,5,6)$ para un $66 \%$ y en los casos 4 y 7 donde determinaba una ausencia de gónadas la laparoscopia encontró su poco desarrollo.

La laparoscopia en últimas para éste grupo confirmó el diagnóstico clínico y sin lugar a dudas la biopsia ovárica evitó una cirugía mayor. A pesar del escaso material para anatomía patológica no debemos desconfiar de la muestra y seguramente una mayor toma de tejido no aporta más para el diagnóstico. En este grupo desde el punto de vista reproductivo su pronóstico es pobre y la respuesta para el desarrollo de la ovulación serán difíciles. A pesar del poco desarrollo genital la terapia de suplencia fue buena (5 casos) 71\% respondiendo con menstruación. En los casos de agenesia uterina (dos casos) la terapia instaurada es discutible con base al tenor de estrógenos encontrados.

Amenorrea Secundaria: Tablas 3.a. y 3.b.

El promedio de edad aquí es mayor 218 años, el tiempo de evolución de su amenorrea es de 2.8. La menarquía se encuentra dentro de los parámetros nor- 
Tabla No. 3.a.

AMENORREA $2 \mathrm{a}$.

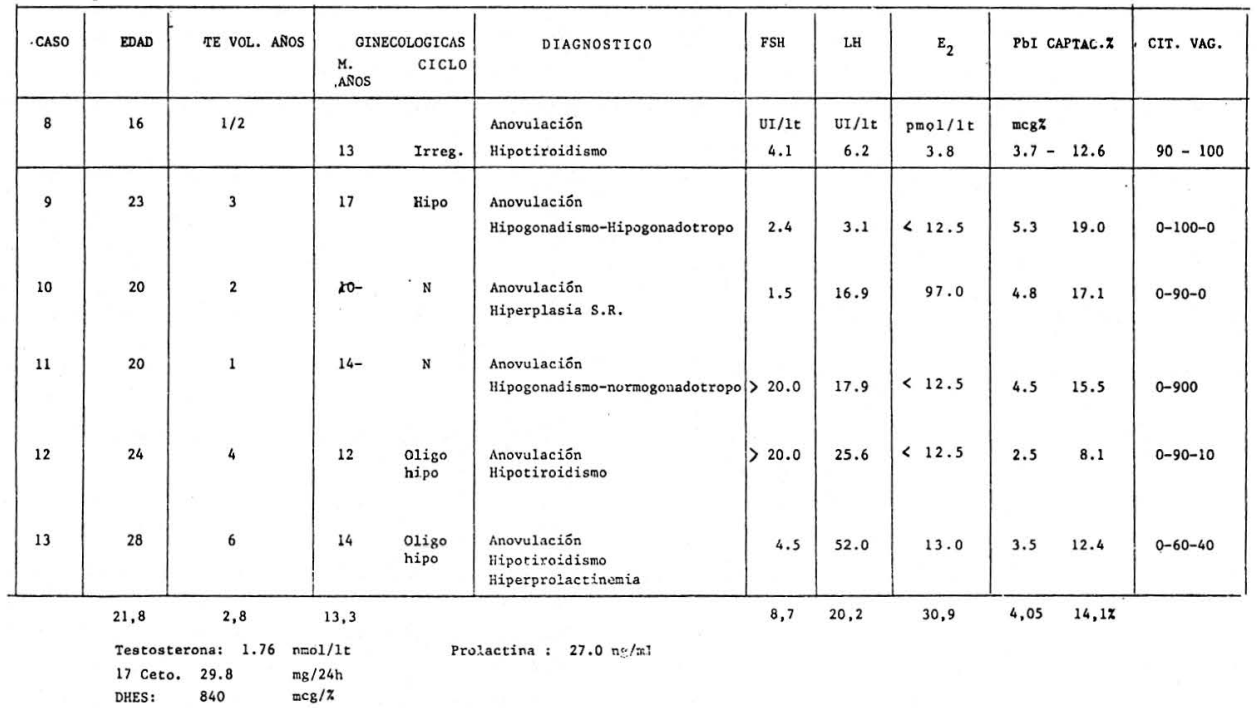

Tabla No. 3.b.

AMENORREA 2a.

\begin{tabular}{|c|c|c|c|c|c|}
\hline CASO & LAPAROSCOPIA & & BIOPSIA & TRATAMIENTO & RESULTADO \\
\hline & UTERO TROMPA & OVARIOS & ALBUGINEA FOLICULOS & & \\
\hline 8 & Hipoplásico & $\begin{array}{l}\text { Crandes } \\
5 \times 4 \times 4 \\
4 \times 3 \times 2\end{array}$ & Eibrosis sin actividad & $P+T x$ & \\
\hline 9 & Hipoplásico & $\begin{array}{l}\text { Normal } \\
3 \times 1 \times 1 \text { 1iso } \\
2 \times 0.4 \times 1\end{array}$ & Fibrosis Ausente & $\mathrm{P}$ & \\
\hline 10 & Hipoplásico & $\begin{array}{l}\text { Grandes } \\
\begin{array}{l}5 \times 3 \times \\
4 \times 3 \times 3\end{array} \\
4 \times 3 \text { liso }\end{array}$ & $\begin{array}{ll}\text { Gruesa } & \begin{array}{l}\text { Normales } \\
(\leftarrow)\end{array}\end{array}$ & $\begin{array}{l}\text { Corticoides } \\
\text { Ciproterona }\end{array}$ & . \\
\hline 11 & Normal & $\begin{array}{l}\text { Hipoplásico } \\
5 \times 0.6 \times 0.11 \text { iso }\end{array}$ & Fibcosis Ausente & $\varepsilon+p$ & \\
\hline 12 & Norma 1 & $\begin{array}{l}\text { Norma: } \\
3 \times 1 \times 1 \\
3 \times 2 \times 2 \quad 2 \text { iso }\end{array}$ & Fibrosiz sin actividad & $\begin{array}{l}E+P \\
+T x\end{array}$ & \\
\hline 13 & Normal Obstrucción & $\begin{array}{l}\text { Hipoplá.ico } \\
2 \times 1 \times 0.5\end{array}$ & Fiorosis oin sictividad & $\underset{S}{\mathrm{~B}} \mathrm{~S}+\mathrm{E}+\mathrm{P}$ & \\
\hline
\end{tabular}

\footnotetext{
$E=$ Estrogeno $\quad \mathrm{P}=$ Progestägeno $\quad \mathrm{TX}=$ Tiroides $\mathrm{B}=$ Bromoergocriptina

s. = Salpingolisis
} 
males 13.3 años y llama la atención la irregularidad menstrual que cursa con hipomenorrea y oligomenorrea (casos 8, $9,12,13)$ para un $66 \%$. En este grupo se muestra una pobre función ovárica con una tasa de estradiol menor de 15 pico moles/litro (casos 9, 11, 12, 13) que se confirma con la citología vaginal funcional.

Si bien la gonadotrofinas se encuentran en el límite normal casos $(11,12$, 13) no queda claro el caso No. 9 que cursa con hipogonadotropismo normogonadotropo a pesar de la ID. de hiperplasia suprarrenal. En los casos de amenorrea secundaria también aparece la asociación de 2 casos con hipotiroidismo. (33\%). El caso 13 es interesante porque cursa con prolactina alta, hipotiroidismo, asociado a hipoplasia ovárica.

En este grupo la laparoscopia despeja el panorama confirma la hipoplasia uterina no sospechada por la clínica y a diferencia del primer grupo, los ovarios aparecen aumentados de tamaño, (casos $8,10)$ normales (casos 9, 12), hipoplásicos $(11,13)$. La anatomía patológica confirma una fibrosis de la albuginea 5 casos (83\%) y a pesar de que aparecen los folículos éstos se encuentran en poca actividad (casos 8, 12, 13) ausente (casos 9 y 11). El tratamiento fue especifico en el caso 13 hiperprolactinemia, el caso 10 de hiperplasia suprarrenal, hipotiroidismo $(8,12)$. La respuesta al tratamiento de suplencia se puede catalogar de buena.

\section{Trastornos de Ciclo: Tablas 4.a. y 4.b.}

Para éste grupo incluimos un caso que llegó externo de una paciente postmenopáusica (caso 18) en quien se sospechaba un tumor virilizante de ovario, por ecografía un tumor de ovario y que en la laparoscopia demostró un hidrosalpinx bilateral y una hipoplasia ovárica. A excepción de éste caso el promedio de

Tabla No. 4.a.

TRASTORNOS DEL CICLO

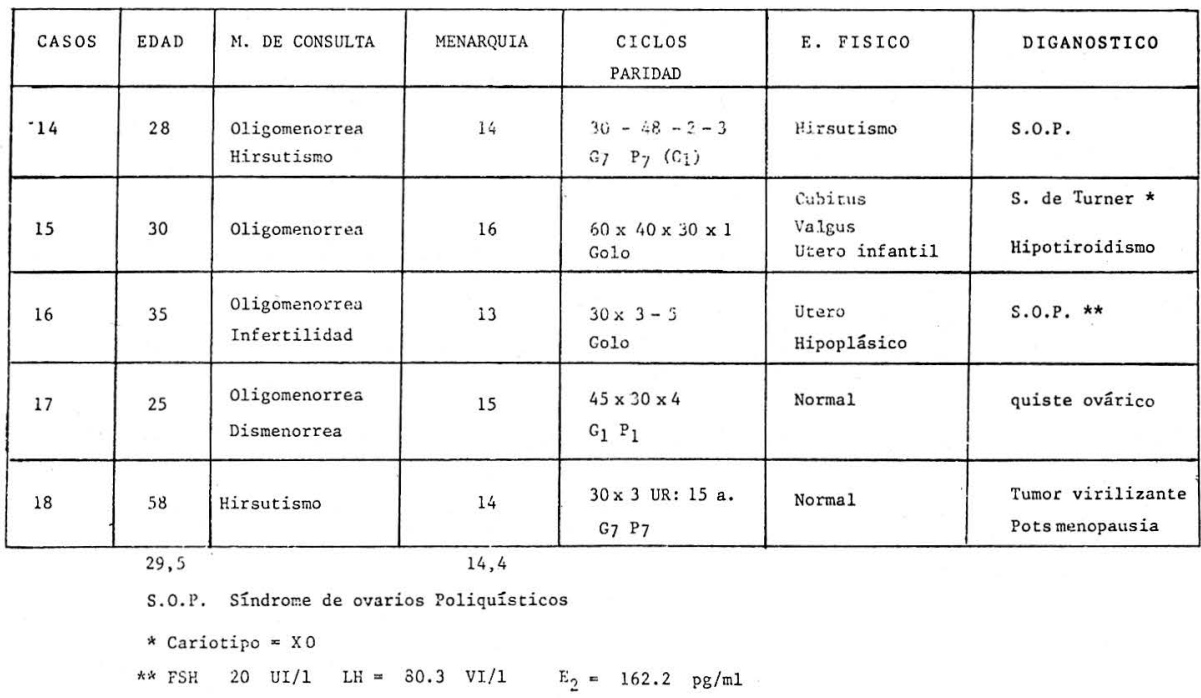


Tabla No. 4.b.

TRASTORNOS DEL CICLO

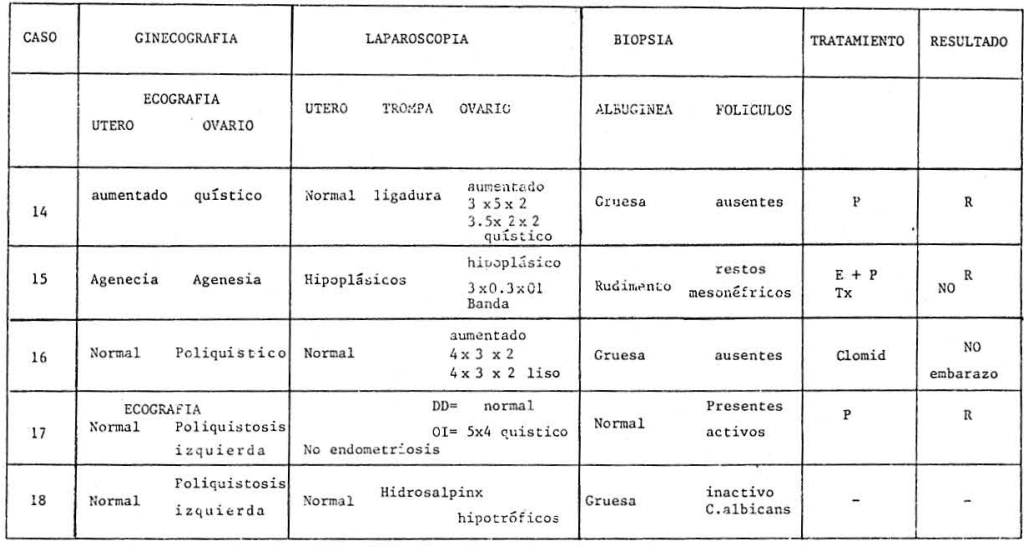

$E=$ Estrógeno $\quad P=$ Progestágeno $\quad T x$ Tiroides

$R=$ rapularizarión menscrual.

edad fue 29.5 años y su motivo de consulta la oligomenorrea. La menarquía fue 14.5 promedio y los ciclos en general fueron largos (40-60 × 2-3 días).

La correlación ginecográfica o ecografía se confirma por laparoscopia el aumento de los ovarios (Síndrome de ovarios poliqu ísticos) casos 14,16 y 17 . La anatomía patológica confirma la albuginea gruesa y la escasez de folículos. El tratamiento discutible específico establece la regularización del ciclo. El caso 15 merece mención especial porque se trata de un síndrome de Turner que a pesar de útero y ovario rudimentarios! menstrua, explicable por tratarse de uná variedad mosaico.

Infertilidad Primaria: Tablas 5.a. y 5.b.

Para este grupo la patología varía hacia la causa básica en nuestro medio de la infertilidad como la lesión tubárica. El promedio de edad fue de 29.3 años y el tiempo de evolución de la infertilidad 3,7 años. La menarquía permanece dentro del promedio 13.7 años. Los ciclos menstruales presentan una tendencia, muy irregular que se confirma por los exámenes paraclínicos como la citología, biopsia endometrial y tasa de progesterona plasmática. Dentro del diagnóstico definitivo encontramos que la obstrucción tubárica o lesión inflamatoria se encuentra en 10 de los 15 casos (66\%) y la lesión ovárica como hipofunción se presenta en 12 casos ( $80 \%$ ). La forma combinada de lesión tubárica y ovárica 9 casos $(60 \%)$. A pesar de ser una casuística no muy extensa planteará en la discusión estos hallazgos. Al correlacionar la citología vaginal funcional, la biopsia de endometrio y la progesterona plasmática determinadas el día 18 y 24 del ciclo, demuestran que son de gran ayuda diagnóstica y como se muestra en la tabla 5.a., en los 8 casos (53\%) con endometrio proliferativo la progesterona plasmática en las dos tomas permanece baja excepto en el caso 11 donde la segunda toma se encuentra en los límites de la curva normal. En los casos (2, 8, 15), donde el endometrio mostró una pobre estimulación es explicable en parte por la baja tasa plasmática de proges 
INFERTILIDAD 1a.

\begin{tabular}{|c|c|c|c|c|c|c|c|c|}
\hline Caso & Edad & Evol. & $\begin{array}{l}\text { Ginecológicas } \\
\text { M. Cíclos }\end{array}$ & Diagnóstico & EV.F & $\begin{array}{l}\text { Bx } \\
\text { Endometrio }\end{array}$ & $\begin{array}{l}\text { Progesterc } \\
18^{\circ}\end{array}$ & \\
\hline 1 & 30 & 1 & $1435 x-3-4$ & $\begin{array}{l}\text { S.O.P. } \\
\text { Obst - tubar. }\end{array}$ & $40-60-0$ & Proliferativo & 12.1 & 240.01 \\
\hline 2 & 32 & 10 & $13 \quad 32 x-4-8$ & $\begin{array}{l}\text { S.O.P. } \\
\text { Obst. tubar }\end{array}$ & $20-50-30$ & Mixto & 195.02 & 270.07 \\
\hline 3 & 22 & 2 & $1435 x-40 \times 2$ & Hiperprolactinemia & $10-70-20$ & $\begin{array}{l}\text { Secretor } \\
\text { pobre }\end{array}$ & 520.7 & 1.022 .0 \\
\hline 4 & 28 & 4 & $12 \quad 24 \times 22 \times 4$ & $\begin{array}{l}\text { C. monofásicos } \\
\text { Obst. tubárica }\end{array}$ & $10-90-0$ & Proliferativo & 198.7 & 440.9 \\
\hline 5 & 30 & 2 & $1230 \times 40 \times 2$ & $\begin{array}{l}\text { C. monofásicos } \\
\text { uterodidelfo }\end{array}$ & $5-90-0$ & No material & 25.0 & \\
\hline 6 & 34 & 4 & $1535 \times 2$ & C. monofásico & $5-90-5$ & Proliferativo & 11.94 & 61.18 \\
\hline 7 & 29 & 6 & $1425 \times 35 \times 2$ & $\begin{array}{l}\text { Poliquistosis } \\
\text { Obst. tubárica }\end{array}$ & $10-90-0$ & Proliferativo & 25.0 & \\
\hline 8 & 34 & 2 & $12 \quad 32 \times 35 \times 3$ & $\begin{array}{l}\text { C. monofásicos } \\
\text { Obst. tubárica }\end{array}$ & $10-60-30$ & Mixto & 1.278 .63 & 2.190 .73 \\
\hline 9 & 37 & 5 & $1135 \times 40 \times 2$ & $\begin{array}{l}\text { C. monofásicos } \\
\text { Obst. tubárica }\end{array}$ & $30-60-0$ & Proliferativo & 198.0 & 220.7 \\
\hline 10 & 23 & 6 & $1430 \times 3$ & Obst. tubárica & $0-60-40$ & Secretor & 2.501 & 1.065 .0 \\
\hline 11 & 38 & 3 & $16 \quad 25 \times 2$ & C. monofásico & $0-70-30$ & Proliferativo & 530.1 & 1.170 .0 \\
\hline 12 & 37 & 4 & $\begin{array}{ll}15 & 35 \times 8\end{array}$ & Obst. tubárica & $5-70-30$ & Secretor & 1.824 .0 & 2.812 .0 \\
\hline 13 & 28 & 4 & $\begin{array}{ll}15 & 120 \times 4\end{array}$ & Ciclo monofásicos & $0-95-5$ & Proliferativo & 25.0 & \\
\hline 14 & 18 & 1 & $14 \quad 24 \times 24$ & $\begin{array}{l}\text { S.O.P. } \\
\text { Salpingitis }\end{array}$ & $0-90-0$ & Proliferativo & 25.0 & \\
\hline 15 & 20 & 2 & $1560 \times 80 \times 2$ & $\begin{array}{l}\text { S.O.P. } \\
\text { Hipotiroidismo }\end{array}$ & $10-70-20$ & Mixto & 117.4 & 52.1 \\
\hline
\end{tabular}


Tabla No. 5.b.

INFERTILIDAD $1 \mathrm{a}$.

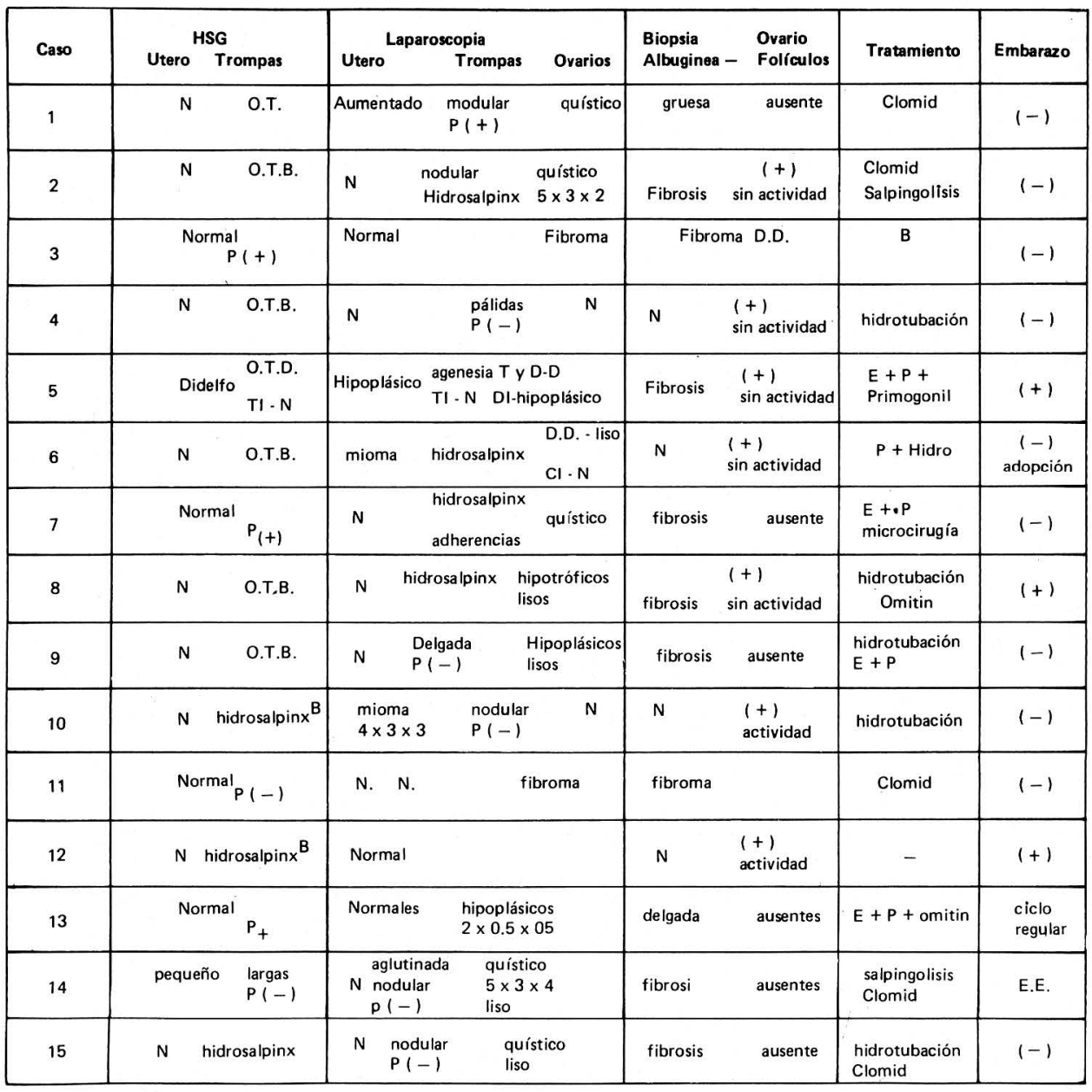


terona pero no en el caso No. 8 que se encuentra dentro de límites normales. Cuando hay un endometrio secretor (casos 10 y 12) tanto la citología como la progesterona están dentro de los parámetros descritos clásicamente.

Al comparar la histerosalpingografía frente a la laparoscopia, no hay duda de la mejor información presentada por la laparoscopia de los órganos pélvicos, analiza la conformación anatómica del útero, trompas, ovarios, tamaño, función, tipo de adherencias y pronóstico de su futuro reproductivo. La correlación de los hallazgos laparoscópicos con los exámenes paraclínicos y las biopsias de ovarios son sorprendentes, pues a diferencia de las lesiones encontradas en las amenorreas, en este grupo si hay folículos pero inactivos que se confirman con todos los exámenes paraclínicos. Los tratamientos efectuados de acuerdo a la lesión están bien indicados y la tasa de embarazo, 4 casos (26.6), incluyendo un ectópico, se encuentra dentro de las estad ísticas mundiales.
Infertilidad Secundaria: Tablas 6.a. y 6.b.

Este grupo no deja de ser interesante pues a pesar de que la edad promedio es de 31.2 años, el tiempo de evolución para llegar a la consulta es más prolongado que en la primaria y aquí es de 6.7 años. La menarquía 12.7 años y como diagnóstico definitivo todos los casos (100\%) cursaron con lesión tubárica. Curiosamente 3 casos $(20,21,22)$ ten ían antecedentes de embarazo ectópico (42.8\%). Como se demuestra por la citología vaginal funcional, la biopsia de endometrio y la tasa de progesterona plasmática los casos 16, 17, 18, 21, ovularon (57.1\%). Los endometrios proliferativos y mixtos cursan con una tasa baja de progesterona plasmática como es lo usual en estos casos. Al revisar la HSG y la laparoscopia la información sobre la función tubárica es correcta por ambos procedimientos y la biopsia confirmó la buena actividad de los ovarios planteando en este grupo hasta dónde puede ser necesario este procedimiento. El tratamiento instaurado es de

Tabla No. 6.a.

INFERTILIDAD 2a.

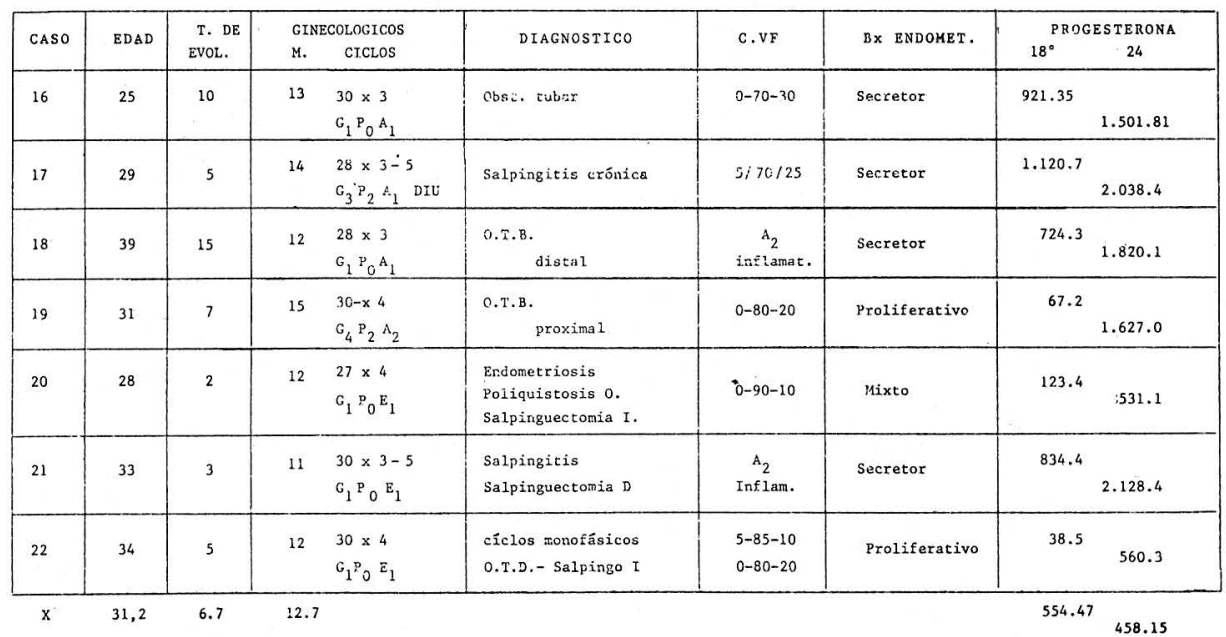


Tabla No. 6.b.

INFERTILIDAD 2a.

\begin{tabular}{|c|c|c|c|c|c|c|c|c|c|}
\hline CASO & UTERO & $\begin{array}{ll}H S & G \\
& T R O M P A S \\
\end{array}$ & UTERO & $\begin{array}{l}\text { LAPAROSCO } \\
\text { TROMRAS }\end{array}$ & $\begin{array}{l}P \text { I A } \\
\text { OVARIO }\end{array}$ & $\begin{array}{r}\text { BIOPSIA } \\
\text { ALBUGINEA } \\
\end{array}$ & $\begin{array}{c}\text { OVARIO } \\
\text { FOLICULOS } \\
\end{array}$ & TRATAMIENTO & EMBARAZO \\
\hline 16 & N. & $\begin{array}{l}\text { Hidrosalpinx } \\
\text { Distal }\end{array}$ & N. & $\begin{array}{l}\text { Pálida } \\
\text { Nodular } \\
P(-)\end{array}$ & $\begin{array}{c}\mathrm{N} . \\
\text { Rugoso } \\
\end{array}$ & N. & $\begin{array}{c}(+) \\
\text { Activos }\end{array}$ & Salpingolisis & $(+)$ \\
\hline 17 & N. & $\begin{array}{l}\text { Obstr. Tubar } \\
\text { Distal }\end{array}$ & N. & $\begin{array}{l}\text { Nodulor } \\
\text { Aglutinadas } \\
R(+)\end{array}$ & $\begin{array}{c}N . \\
\text { Rugoso } \\
\end{array}$ & N. & $\begin{array}{l}(+) \\
\text { Activos } \\
\text { C. Amaril10 } \\
\end{array}$ & $\begin{array}{l}\text { Antisioticos } \\
\text { Antinfiamatorio }\end{array}$ & $(+)$ \\
\hline 18 & N. & Hidrosalpinx & $\begin{array}{l}\text { Aumen } \\
\text { tacoo }\end{array}$ & $\begin{array}{l}\text { Hidrosalpin } x \\
5 \times 2 \times 2\end{array}$ & N. & N. & $\begin{array}{c}(+) \\
\text { Escascs } \\
\text { Activos } \\
\end{array}$ & Salpingolls is & $\begin{array}{c}(-) \\
\text { adop }=10 \text { ón }\end{array}$ \\
\hline 19 & N. & $\begin{array}{l}\text { Obstr. "ubar } \\
\text { Proximal }\end{array}$ & N. & $\begin{array}{l}\text { Nodular } \\
\text { Aglutinada }\end{array}$ & $\begin{array}{l}\text { Pollquis } \\
\text { tico } \\
3 \times 5 \times 2 \\
\end{array}$ & Delgada & $\begin{array}{l}\quad(+) \\
\text { Inactivos } \\
\text { Qulstes }\end{array}$ & Reanastonosis 0 & $(-)$ \\
\hline 20 & N. & $\begin{array}{l}\text { HIdrosaipinx } 0 \\
\text { O.T. Izquierda }\end{array}$ & N. & 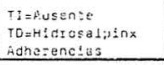 & $\begin{array}{l}\text { Poliquí: } \\
\text { t:eo }\end{array}$ & Deigada & $\begin{array}{l}(+) \\
\text { Escasa Acti- } \\
\text { vlaad }\end{array}$ & $\begin{array}{l}\text { Hidrotubaclón } \\
\text { Salpingollsis }\end{array}$ & $(-)$ \\
\hline 21 & & Este no se reallzo & N. & $\begin{array}{l}\text { TO=Alisente } \\
T I=\text { Aglutinada } \\
P(-) \text { Adherenclas }\end{array}$ & N. & N. & $\begin{array}{c}(+) \\
\text { Escasos } \\
\text { Activos } \\
\end{array}$ & $\begin{array}{l}\text { Hiorctudacín } \\
\text { Salpingolisis }\end{array}$ & $(-)$ \\
\hline 22 & N. & $\begin{array}{l}\text { Oostr. Tubas. } \\
\text { Proximal }\end{array}$ & $\mathrm{N}$. & $\begin{array}{l}\text { TO=Ausante } \\
T I=\text { Nodula: } \\
\text { P(-) Rehezencios }\end{array}$ & $\begin{array}{l}O D=4 \times 5 \times 3 \\
O \mathrm{I}=\mathrm{N} .\end{array}$ & N. & $\begin{array}{l}\quad(+) \\
\text { Escesa } \\
\text { fetiviuas }\end{array}$ & $\begin{array}{l}\text { Hidrotubación } \\
\text { Inductotes } \\
\text { Salpingoilsis }\end{array}$ & $(-)$ \\
\hline
\end{tabular}

discusión, pero con el avance de la microcirugía la tasa de embarazo deberá ser más alta que en la primaria. Con dos embarazos en este giupo (28.5\%) está dentro del promedio pero susceptible de mejorar.

\section{DISCUSION:}

Si bien la controversia sobre la utilidad o no de la biopsia de ovario por laparoscopia para algunos autores mostraba restricciones (5), el presente trabajo demuestra la utilidad como complemento diagnóstico en amenorrea primaria, secundaria, trastornos del ciclo e infertilidad primaria. Si resumimos los hallazgoz encontrados como se muestran en la tabla 7 podemos ver como la patología ovárica se proyecta desde su diagnóstico laparoscópico de hipoplasid, la que se confirma por la biopsia y donde el clínico espera más y no va a encontrar más, de acuerdo con los estudios de otros autores $(6$ y 7$)$. En amenorrea secundaria ya aparecen estos folículos pero su actividad es limitada pero su pronóstico es mejor. Los trastornos del ciclo conducen a estudiar un poco más la poliquistosis ovárica que seguramente será una patología abundante en este grupo.

La infertilidad a pesar de la escasa casuística determina dos grupos muy claros, la infertilidad primaria asociada no sólo a una lesión tubárica sino agravada por hipofunción ovárica (poliquistosis), el segundo grupo compuesto por infertilidad secundaria donde predomina la lesión tubárica y que nos plantea la posibilidad de no hacer biopsia si se tienen datos que confirman la ovulación como la CVF, biopsia endometrial, progesterona plasmática y ecografía (7) y 11).

Los hallazgos en el grupo de amenorrea primaria nos plantea una duda sobre si se debe hacer biopsia en los casos donde se encuentra una banda o "Streak Gonadal". Algunos autores informan que no debe hacerse por el peligro de lesionar el útero (4). A pesar de ésto, en otros 
Tabla No. 7

RESUMEN DE BIOPSIA DE OVARIO POR LAPAROSCOPIA

\begin{tabular}{|c|c|c|c|c|c|c|c|}
\hline CLASIFICACION & EDAD & T. DE EVOLUCION & DIAGNOSTICO & LAPAROSCOPIA & BX OVARIO & тTо & RESULTADO \\
\hline Amenorrea primaria & 19 & $4-5$ & $\begin{array}{l}\text { Hipoplasia } \\
\text { utero ovárica }\end{array}$ & $\begin{array}{l}\text { Hipoplasia } \\
\text { ovấrica }\end{array}$ & $\begin{array}{l}\text { fíbrosis } \\
\text { gónada }\end{array}$ & $\begin{array}{l}\text { estrogen. } \\
+ \text { progest. }\end{array}$ & menstruacion. \\
\hline Amenorrea secundaria & 21.8 & 3.5 & $\begin{array}{l}\text { disfunciôn. } \\
\text { ouârica }\end{array}$ & $\begin{array}{l}\text { Hipoplasia } \\
\text { ovárica }\end{array}$ & $\begin{array}{l}\text { folículos } \\
\text { escasos }\end{array}$ & $\begin{array}{l}\text { proges tá- } \\
\text { genos. }\end{array}$ & " \\
\hline cíclo irregular & 29.5 & - & $\begin{array}{l}\text { Poliquistosis } \\
\text { ovärica }\end{array}$ & Poliquistosis & $\begin{array}{l}\text { Albuginea } \\
\text { gruesa, foli- } \\
\text { culos escasos }\end{array}$ & Inductores & $"$ \\
\hline I. Primaria & 29.3 & 3.8 & $\begin{array}{l}\text { Obstrucción tubá- } \\
\text { rica + disfunciōn } \\
\text { ovărica }\end{array}$ & $\begin{array}{l}\text { Obst. tubárica } \\
+ \text { poliquist. }\end{array}$ & $\begin{array}{l}\text { Albuginea } \\
\text { gruesa, fo- } \\
\text { lículos es- } \\
\text { casos. }\end{array}$ & $\begin{array}{l}\text { Hidrotuba- } \\
\text { ción } \\
\text { Microcirugia } \\
\text { Inductores }\end{array}$ & $\begin{array}{l}\text { Embarazo } \\
28 \%\end{array}$ \\
\hline I. Secundaria & 31.2 & 6.7 & Obst. tubärica & $\begin{array}{l}\text { Adherencias } \\
+ \text { Obst. tub. }\end{array}$ & Normal & $\begin{array}{l}\text { Hidrotuba- } \\
\text { ciones, } \\
\text { microcirug. }\end{array}$ & $\begin{array}{l}\text { Embarazo } \\
28.5 \%\end{array}$ \\
\hline
\end{tabular}

estudios $(7,8)$ los investigadores proponen como alternativa pronóstico antes de llevar a una laparotomía pues en estos casos como sucede en el testículo, la activación de éstos folículos es difícil por el daño previo sobre sus receptores. La amenorrea secundaria por el contrario su pronóstico mejora por los datos encontrados no sólo en los cortes histológicos sino por la proporción más alta de los esteroides (10).

En los casos de infertilidad primaria si bien puede agravar el pronóstico, una revisión reciente demuestra cómo en los casos de poliquistosis ovárica la sola biopsia puede ser útil para mejorar la condición del ovario (12). Nos quedan serias dudas sobre si se debe hacer biopsia en infertilidad secundaria cuando

\section{BIBLIOGRAFIA}

1. STEEL S.J. BEILBY J.W., PAPADAKI L; Visualization and biopsy of the ovary in the investigation of amenorrhea. Obstet. Gynecol. 36; 899-902.

2. NEUWIRTH R.S., A method of bilateral paraclínicamente se conoce la buena función de ovario. En este caso sólo cuando se sospeche patología ovárica se recomienda la biopsia (poliquistosis ovárica).

Este estudio demuestra una vez más que al clasificar las diferentes entidades ginecológicas donde un procedimiento aporta o complementa el diagnóstico puede ser útil. No nos podemos dejar engañar con la primera impresión de un trabajo donde los resultados fueron deficientes. Debemos, sin embargo, en nuestro medio, tener en cuenta tales apreciaciones y con éste pequeño trabajo confirmar el falso concepto sobre la poca utilidad de la biopsia ovárica por laparoscopia. ovarian biopsy at laparoscopy in fertility and cronic anovulation. Fertil Steril 1972,$23 ; 361-366$.

3. SYKES D.W. GINSBURG J. The use of laparoscopic ovarian biopsy to assess gonadal function. Am. J. Obstetric Gynecol. $1972,112,408413$. 
4. FAYEZ J.A., JONAS HS, Assessment of the role of laparoscopic ovarian biopsy. Obst. Gynecol. 1976; 48, 397-402.

5. PORTUONDO J.A. NAVARRO E, BENITO J.A. OBREGON M.J. Indications for and limitations of laparoscopic ovarian biopsy. J. Reprod. Med. 1982: 27 (2), 67-62.

6. CORSON SL. Use of the laparoscopic in the fertil patient. Fertil Steril 1979: 32 (4) 359-369.

7. ARQUEROS J. NAVARRO J., CARRASCO F., JUAN J., SANTAOLAYA A. Utility of laparoscopy associated to the ovarian biopsy in the study of gonadal function. Rev. Esp. Obstet. Gynecol. $1979 ; 38,165-171$.

8. BATEMAN B.G., NUNLEY WC. JR., KITCHIN JD, Sd. Hipergonadotropic ovarian failure in young women. Int. $\mathbf{J}$. Gynecol. Obstet. 1983 21;305-14.

9. BERECK J.S. HACKER NF. Laparosco- py in the managment of patients with the ovarian carcinoma. Clin. Obstet. Gynecol. $1983-10 ; 213-222$.

10. PORUONDO J.A. TEJADA RF, BENITO JA, BILBAO FG. Laparoscopy, ovarian and endometrial biopsies in secondary amenorrhea. Endoscopy 1982 14. 209. 211.

11. JONES HW, ROCK JA. Repartive and constructive surgery of the female generative tract. William Wilkins, Baltimore 1983.

12. CONEY P. Polycystic ovarian disease current concepts of pathophysiology and therapy. Fertil Steril 1984, 42: 667 682.

13. ADASHI EY, ROCK JA. GUZICK D. WENT AC, JONES GS. JONES HW Jr. Fertility following bilateral ovarian wedge resection: A critical analysis of 90 consecutives cases of the polycystic ovary sindrome. Fertil Steril 1981, 36: 320 325 . 